Compte rendu de thèse

\title{
Ludovic Falaix, Des vagues et des hommes. La glisse au cou des résistances et contestations face à l'institutionnalisation des territoires du surf en Aquitaine
}

Thèse de doctorat en aménagement-géographie, Université de Pau et des Pays de l'Adour, dirigée par Vincent Vlès

(soutenue le 6 juin 2012)

\section{Ludovic Falaix}

\section{(2) OpenEdition}

\section{Journals}

Édition électronique

URL : http://journals.openedition.org/tourisme/250

DOI : 10.4000/tourisme.250

ISSN : 2492-7503

\section{Éditeur}

Éditions touristiques européennes

\section{Édition imprimée}

Date de publication : 1 décembre 2012

Pagination : 102-105

ISSN : 2109-5671

\section{Référence électronique}

Ludovic Falaix, « Ludovic Falaix, Des vagues et des hommes. La glisse au cœur des résistances et contestations face à l'institutionnalisation des territoires du surf en Aquitaine », Mondes du Tourisme [En ligne], 6 | 2012, mis en ligne le 30 septembre 2015, consulté le 22 septembre 2020. URL : http:// journals.openedition.org/tourisme/250 ; DOI : https://doi.org/10.4000/tourisme.250

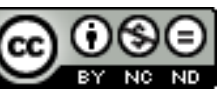

Mondes du tourisme est mis à disposition selon les termes de la licence Creative Commons Attribution - Pas d'Utilisation Commerciale - Pas de Modification 4.0 International. 


\title{
COMPTE RENDU DE THÈSE
}

\section{Des vagues et des hommes}

\section{La glisse au cœur des résistances}

\section{et contestations face à l'institutionnalisation des territoires du surf en Aquitaine}

\author{
Thèse de doctorat en aménagement-géographie, Université de Pau et des Pays de l'Adour \\ dirigée par Vincent Vlès \\ (soutenue le 6 juin 2012)
}

LUDOVIC FALAIX_ [ludovicfalaix@yahoo.fr]

E

n s'interrogeant sur la manière dont les surfeurs habitent l'espace-vague, cette thèse s'attache à construire comme énigme de recherche l'observation de leurs résistances et contestations face à l'institutionnalisation de leurs territoires de pratique. Elle mobilise l'habiter, entendu comme un nouveau concept riche en controverses scientifiques (Frelat-Kahn et Lazzarotti, 2012; Berque, de Biase et Bonnin, 2008; Goetz, 2011; Paquot, Lussault et Younès, 2007), afin d'élaborer une géographie de l'intime présentée comme l'étude de la dimension affective que les hommes convoquent pour se spatialiser.

L'examen de l'apport des travaux sur le surf et les surfeurs réalisé dans la thèse (Augustin, 1994 ; Loret, 1995 ; Guibert, 2006 ; Sayeux, 2008) souligne une lacune récurrente relative à la saisie du caractère intime et émotionnel du rapport vécu du surfeur à la vague. À partir d'une observation participante et d'une proximité relationnelle établie auprès des surfeurs interrogés, cette thèse met en lumière le fait, qu'à travers l'acte de glisse, les surfeurs agissent sur la vague en se gratifiant (Laborit, 1974), pratiquent le lieu (de Certeau, 1990) et le métamorphosent ainsi en espace. Ils nourrissent alors le sentiment d'une existence géographique induite par l'habitabilité, la cosmogonisation et la sacralisation de la vague.

Forts de ces résultats, cette recherche distingue donc les termes de lieu-vague et d'espace-vague. Elle détermine que le premier est insignifiant, un chaos, une portion d'espace terrestre a-spatialisante, ou, aussi paradoxal que cela puisse paraître, un "non-lieu” (Augé, 1992), tandis que le second est sacralisé, habité, un cosmos à partir duquel le surfeur éprouve le sentiment d'exister sur terre, ici et maintenant. L'espace n'est alors plus envisagé comme déjà acquis par l'homme et abordé comme seul support de ses spatialités. La spatialisation est alors définie comme l'habitabilité des lieux, et non des espaces, comme un processus induit par le fait de se sentir "affecté en affection" (Zubiri, 1962) par le "génie des lieux" (Pitte, 20।0). L'espace est le fruit de l'action d'un individu restitué dans sa pro- 
fondeur, son intimité, sa sensibilité, son émotion, sa poï̀sis, sa capacité à intérioriser les informations issues de son environnement sociogéographique. Ainsi, la géographie de l'intime appréhende "l'intelligence sentante" (Zubiri, 1962) des individus, leur "arkhé-pensée" (Morin, 1986) comme des outils au service de la compréhension de la manière dont les hommes se spatialisent, produisent leurs espaces (Lefebvre, 2000), c'est-à-dire établissent "leurs sphères” (Sloterdijk, 1998), protègent leurs "espaces louangés" (Bachelard, 1957), sécurisent leur existence "dans et par l'écoumène" (Berque, 2000).

En accord avec le fait que "les espaces reçoivent leur être des lieux et non de 'L'espace'” (Heidegger, 1954), cette recherche élabore donc une géographie de l'intime appréhendée comme l'étude de l'espace habité afin d'envisager "la géographicité” (Dardel, 1952) des surfeurs, leurs "prises trajectives" (Berque, 2000), leur "condition géographique” (Lazzarroti, 2006). Elle s'appuie sur l'approche phénoménologique (Hoyaux, 2002) et considère le corps comme un effecteur ontologique dans la mesure où la confrontation au lieu-vague permet au surfeur de prendre la mesure de sa condition géographique. Il accomplit, via la glisse, sa poésie entendue comme "le faire habiter originel” (Heidegger, 1954). Il expérimente le passage de "l'espace spatialisé à l'espace spatialisant" (Merleau-Ponty, 1945). La géographie de l'intime n'est donc pas l'explo- ration de l'espace privé. Elle s'attache plutôt à définir l'espace comme une construction qui dépend de la manière dont les lieux entrent en résonance chez l'individu. En fonction de ces résonances, qui mettent en jeu l'intime et l'affectif, une micro-géographie corporelle, l'individu métamorphose le lieu en espace. Ce n'est qu'une fois cet acte de transformation accompli et opéré que l'individu s'empare de sa condition géographique, prend conscience de son existentialité et occupe, pratique, habite, non pas le lieu préexistant, mais bel et bien l'espace. Ce n'est qu'à partir du moment où le lieu acquiert sa dimension spatialisante que l'espace peut être vécu (Frémont, 1976). Cette pourquoi cette thèse propose également une relecture de la théorie de l'habiter forgée par Mathis Stock (2007) qui préconise de dépouiller l'habiter de sa connotation écologique et cosmologique.

Cette recherche questionne donc le déterminisme social qui serait au fondement de tout acte géographique. Car, si la production de l'espace est la transformation du lieu, la métamorphose du chaos en cosmos (Eliade, 1957), ne conviendrait-il pas mieux de considérer que l'espace serait, de part en part, non seulement sociétal, mais davantage l'expression de la poésie d'individus socialisés (Levy et Lussault, 2003) ? Par extension, cette thèse propose une clé de lecture de l' “acosmie” (Berque, 2008) ou de la déterritorialisation. Ces processus traduiraient un déficit chronique des individus à pouvoir se spatialiser c'est-à-dire à opérer la transformation des lieux en espaces. C'est à l'aune de cette hypothèse que cette thèse examine les résistances et contestations des surfeurs face à l'institutionnalisation des territoires du surf en Aquitaine.

Non sans revenir sur les conditions d'implantation et de développement du surf en Aquitaine, cette recherche étudie comment les pouvoirs publics, forts de la réhabilitation de l'image du surfeur dans le paysage journalistique local image fondée sur une galerie de portraits idéaux typiques (le héros sportif, le philanthrope, le businessman, l'écologiste) - et conscients des enjeux territoriaux inhérents à la promotion de cette pratique sportive, accompagnent l'ancrage du surf et orchestrent l'intégration sociospatiale du surf au sein des espaces urbains de ces stations balnéaires. Cette forme d'institutionnalisation des territoires du surf, déclinée dans les outils de prospective et de planification territoriale, se traduit par "une mise en scène (géo)graphique” (Debarbieux, 2003) du potentiel récréatif du littoral aquitain, par une diversification de l'offre sportive au bénéfice d'un renforcement de l'attractivité touristique (Falaix, 20I2). Le surf devient ainsi progressivement une icône de qualité de vie et de dynamisme par l'ensemble de la communication territoriale. Les néoterritorialités sportives induites par la démocratisation du surf sur la côte aquitaine bouleversent la structuration des 
stations balnéaires fondée sur plus de deux siècles de développement touristique (Falaix et Favory, 2009). L'omniprésence du surf comme marqueur territorial s'accompagne d'un renforcement d'une offre touristico-sportive qui préside à une nouvelle perception du littoral aquitain autour d'un surf policé, sécurisé et plus accessible, au risque d'un lissage de l'offre récréative. Cette assimilation du surf implique une gestion sécuritaire et réglementaire appropriée, une intégration plus large dans une offre associative, scolaire et universitaire et même la création de grands équipements structurants pour conforter la filière surf en Aquitaine. Cette thèse met ainsi en évidence la nature incrémentale du changement des politiques publiques (Muller, 2005) induite par la prise en compte du surf dans l'action publique en faveur du tourisme, de l'aménagement et du développement local, du sport, de l'action sociale...

Toutefois, cette institutionnalisation des territoires du surf n'est pas sans provoquer des résistances et contestations de la part de certains surfeurs. Celles-ci, jusqu'ici présentées comme des luttes intestines et stigmatisées dans le seul champ de la déviance, sont alors interprétées comme les manifesta- tions d'un désir de préserver la dimension ontologique de l'espacevague habité (Falaix, 2009). À l'heure où le surf est mobilisé pour réenchanter le littoral aquitain, certains surfeurs, désenchantés visà-vis d'un tel projet de développement touristique, stigmatisé comme responsable de la surfréquentation des espaces de pratique, résistent et contestent en réponse à ce qu'ils ressentent comme une atteinte à leur existentialité. Au rang de ces modalités de résistances et de contestations figure la mobilisation environnementale, comprise comme un mode de ménagement de l'espace-vague habité. L'exil ou

\section{RÉFÉRENCES BIBLIOGRAPHIQUES}

Marc Augé, Non-Lieux, introduction à une anthropologie de la surmodernité, Seuil, 1992.

Jean-Pierre Augustin, Surf Atlantique : les territoires de l'éphémère, MSHA, 1994.

Gaston BACHelard, La Poétique de l'espace, PUF, [1957], 2001.

Augustin BERQUE, Ecoumène. Introduction à l'étude des milieux

humains, Belin, 2000.

Augustin BERQUE, "Trouver place humaine dans le cosmos", Echogéo, $n^{\circ} 5,2008$.

Augustin Berque, Alessia DE BIASE et Philippe BonNIN, L'Habiter dans sa poétique première : actes du colloque de Cerisy-la-Salle, Donner lieu, 2008.

Michel de Certeau, L'Invention du quotidien. I Arts de faire, Gallimard, 1990.

Éric DARDEL, L'Homme et la terre, nature de la réalité géographique, CTHS, [1952], 1990.

Bernard DebarbieuX, "Neuf enjeux de l'iconographie de projet et de prospective de territoire", dans Bernard DEBARBIEUX et Sylvie LARDON, Les figures du projet territorial, Éditions de l'Aube. 2003.
Mircéa ElIADE, Le Sacré et le profane, Gallimard, [1957], 2002.

Ludovic FalaIX et Michel FAvORY, "Les stations du surf sur la côte

basque", Sud Ouest européen, n 13, 2002.

Ludovic FalaIX, “Une géographie de l'intime. L'exemple des territoires du surf', Revue européenne de management du sport, n²4, 2009.

Ludovic FALAIX, "Les sports de nature dans le département des Landes. Du développement maîtrisé à la mobilisation d'une ressource territoriale", Annales de géographie, n 686, 2012.

Brigitte Frelat-KaHN et Olivier LAZZAROTTI, Habiter, vers un nouveau concept?, Armand Colin, 2012.

Armand Frémont, La Région, espace vécu, Flammarion, [1976], 1999. Benoît Goetz, Théorie des maisons : I'habitation, la surprise, Verdier, 2011.

Christophe GUIBERT, L'Univers du surf et stratégies politiques en Aquitaine, L'Harmattan, 2006.

Martin Heidegger, Essais et conférences, Gallimard, [1954], 200 I.

André-Frédéric Hoyaux, "Entre construction territoriale et constitution ontologique de l'habitant : introduction épistémologique aux 
le trip surf sont explicités comme moyens de prendre des vagues "jusqu'à plus soif”, c'est-à-dire comme un moyen d'explorer l'intensité du caractère ontologique induit par la métamorphose du lieu-vague en espace-vague. Quant aux revendications spatio-identitaires, elles sont ici interprétées comme une modalité de préservation du potentiel spatialisant que suppose l'acte de glisse. Enfin, l'abandon provisoire ou définitif du surf constitue également une forme de résistance à l'institutionnalisation des territoires du surf en Aquitaine. Néanmoins, cet abandon de la pratique du surf s'accomplit au béné- fice de néoterritorialités sportives au sein desquelles ces surfeurs, désabusés par l'instrumentalisation de la culture surf et de leurs espaces de pratique, renouent avec la dimension spatialisante que leur confèrent leurs pratiques sportives accomplies au cour de milieux naturels souvent vierges de toute procédure d'aménagement.

Initialement, cette thèse visait à mobiliser les sciences sociales pour comprendre l'univers du surf. Chemin faisant, elle a souhaité apporter une contribution à la compréhension des pratiques récréatives de nature et à l'approche géographique des loisirs sportifs. La géographie de l'intime est un moyen d'embrasser avec une acuité nouvelle les résistances et contestations territoriales, les prises de risque dans la confrontation aux milieux terrestres, dans la mesure où celles-ci seraient comprises comme des moyens de préserver ou d'éprouver intensément l'existentialité induite par le fait d'habiter l'espace. La géographie de l'intime peut également être un outil dans le cadre d'une déclinaison opérationnelle pour aménager l'espace habité en ménageant le potentiel ontologique que soustend le rapport des hommes aux lieux. apports de la phénoménologie au concept d'habiter", Cybergéo, mis en ligne le 29 mai 2002 [http://cybergeo.revues.org//824].

Henry LABORIT, La Nouvelle Grille, Gallimard, [1974], 2000.

Olivier LAZZAROTtı, Habiter, la condition géographique, Belin, 2006.

Henri LefeBVRe, La Production de l'espace, Anthropos, 2000.

Jacques LÉVY et Michel Lussault (dir.), Dictionnaire de la géographie et de l'espace des sociétés, Belin, 2003.

Alain LORET, Génération glisse : dans l'eau, l'air, la neige... La révolution du sport des "années fun", Autrement, "Mutations", n I55-156, 1995.

Maurice MerLeAU-PONTY, Phénoménologie de la perception, Gallimard, [1945], 2003.

Edgar MoRIN, La Méthode - Tome III : La connaissance de la connaissance, Seuil, [1986], 200।.

Pierre Muller, "Esquisse d'une théorie du changement dans l'action publique : structures, acteurs et cadres cognitifs", Revue française de science politique, vol. 55, n I, 2005.

Thierry PAQUOT, Michel Lussault et Chris YounĖs (dir.), Habiter, le propre de l'humain. Villes, territoires et philosophie, La Découverte,

2007.
Jean-Robert PITte, Le Génie des lieux, CNRS Editions, 2010.

Anne-Sophie SAYEUX, Surfeurs, l'être au monde. Une analyse socioanthropologique, Presses universitaires de Rennes, 2008.

Peter SLOTERDIJK, Bulles. Sphères I, Hachette Littérature, [1998], 2002. Mathis STOCK, "Théorie de l'habiter. Questionnements", dans Thierry Paquot, Michel Lussault et Chris Younès (dir.), Habiter, le propre de l'humain. Villes, territoires et philosophie, La Découverte, 2007.

Xavier ZuBIRI, Traité de réalité, L'Harmattan, [1962], 2010. 